\title{
Using Persona as Lenses for a Reference Model
}

\author{
Aurora Constantin \\ University of Edinburgh \\ Edinburgh, EH8 9AB, UK \\ Aurora.Constantin@ed.ac.uk \\ Abraham Nieva \\ Cardiff University \\ Cardiff, CF24 3AA, UK \\ NievadelaHidalgaA@cardiff.ac.uk
}

\author{
Alex Hardisty \\ Cardiff University \\ Cardiff, CF24 3AA, UK \\ HardistyAR@cardiff.ac.uk
}

\begin{abstract}
This paper investigates the value of persona in relation to a conceptual product: the ENVRI reference model designed for environmental research infrastructures. Three personas have been created to understand the use of the model and the challenges faced when applying it. Personas helped identify the level of support required by different users, prioritise the audience to address first, and revealed what aspects of the model are important to different audiences. We have made significant progress in understanding how to improve communication about the model to each persona.
\end{abstract}

\section{Personas, User-centred Design, Reference Models, Environmental Research Infrastructures}

\section{INTRODUCTION}

The development of environmental research, mainly driven by global critical challenges, such as climate change or destruction of natural resources, has led to an unprecedented complexity of the Research Infrastructures (RIs) and growth of data collection. In this context, the ENVRI Reference Model (RM) has been developed to help Rls cope with their complexity and to facilitate interoperability and resource sharing across environmental science domains [1].

The definition of research infrastructure adopted in this paper comes from the European Strategy Forum of Rls: "Research Infrastructures are facilities, resources or services of a unique nature that have been identified by European research communities to conduct top-level activities in all fields" [2, p. 10]. For example, EUFAR (European Facility for Airborne Research) is an RI, focused on the airborne research in the environmental and geo-sciences domains [3].

An RM is a descriptive conceptual framework, establishing a common language of communication and understanding about elements of a system and their significant relationships, within a community of interest $[4,5]$. For instance, the Reference Model for Open Distributed Processing (RM-ODP) has been used for describing space data systems [6], government enterprise architectures [7], and environmental information infrastructures [8].

Despite the RM being successfully applied [9] and the efforts made to improve Rls engagement (including the development of a set of tutorials [10]), the RM is not used as much as expected. During an ENVRI week in Prague, autumn 2016, many ideas were generated by technical experts to facilitate the engagement of more RIs with the RM. These ideas revealed the demand for improving the communication about the RM to its various users.

We adopted persona, a popular technique in Usercentred Design (UCD), to allow the users' needs, goals and challenges to drive the design of possible ways to better communicate the RM. Typically, a persona is a representation of a specific audience segment for a product or a service, and embodies users' needs, goals and challenges [11, 12].

This paper describes the research work of investigating the value of persona in relation to a conceptual product: the ENVRI RM. The aim is to improve the communication about the RM to its users within the environmental RIs in order to increase its adoption.

\section{PREVIOUS WORK}

This section will be focused on the research literature relevant to reference models, specifically the ENVRI RM, as well as that regarding the persona technique which has been chosen to approach the research question.

\subsection{ENVRI RM overview}

The ENVRI RM is based on the RM-ODP [4], that provides a conceptual framework for complex business-oriented systems and helps structure their 
specification. Consequently, the ENVRI RM is defined from five different viewpoints: science (corresponding to the business viewpoint in the RM-ODP), information, computational, engineering and technology [1].

The ENVRI RM is intended to help develop understanding and encourages people to take a consistent approach. Taken together the five viewpoints offer a rich description of the system, but researchers can assess whether their interests are covered by studying only a subset of those viewpoints. They also facilitate knowledge and expertise sharing by providing different perspectives that can be adopted according to a user's set of skills. At the same time, they are consistent with each other.

\subsection{Persona technique}

Cooper and colleagues introduced personas to help designers understand users' motivations, skills, attitudes and goals [12]. Personas capture the patterns of real users' behaviours. These patterns are identified from data collected through ethnographic methods, such as interviews, observations, or questionnaires. Though personas are fictional characters, their roots in real data endow them with the power of empathy. They enable designers to empathise with the users, and make fewer wrong decisions [12].

Although personas have been used for many purposes $[13,14,15,16]$, we have not found work focusing on how best to present a conceptual framework. The intent of the ENVRI RM is to encourage systems thinking, so that decision makers can steer their systems to be compatible and share effort. Thus, the ENVRI RM should develop understanding of the relevant abstract structures. This paper investigates the value of persona for such a conceptual goal. Since this problem is general, and difficult to be fully addressed within the temporal limits of a project, the following research question has been derived to be addressed:

$R Q$ : Can the persona technique be helpful for a specific reference model (i.e. ENVRI RM)?

The remainder of this article will attempt to answer this research question which refers to one sample conceptual RM in one community. However, we consider that this is an important step in taking further the persona technique and applying it for conceptual products.

\section{METHODS}

A series of semi-structured interviews, observations, online surveys, questionnaires and a brainstorming session were employed. These were conducted in four stages (Fig. 1) by the first author, in various research institutions, during expert meetings, as well as during the fourth ENVRI week.

First, a set of 7 people were interviewed using a semi- structured approach, during an expert meeting focused on applying the RM for a group of Rls in the atmospheric domain. Over the 3-day meeting, 10 people were observed while using the RM for their RIs. Second, another round of similar 12 semi-structured interviews were conducted during a meeting focused on using the RM for marine domain Rls. At the same time, observations of the 15 participants working with the RM were made over the 2-day meeting. Third, during the fourth ENVRI week, three developed personas were presented to 40 participants in a workshop. Then, they were asked to fill in a questionnaire about which persona best matched them. At the end of the workshop, the participants were invited to write their suggestions for improving the communication about the RM for the persona they identified with on paper notes. 20 questionnaires were returned with the paper notes.

The decision was taken to focus on $\mathrm{RI}$ professional persona and to develop a series of consultations (modules presenting an aspect of the RM and asking related questions). The first module (an overview of the RM) was presented to 20 people, at a workshop during a meeting in a solid Earth research institution. Questionnaires (with the RI professional description) were used to collect feedback. The aims were to better understand whether the overview is appropriate for the targeted persona, and to collect suggestions for improving it. Six questionnaires were returned.

Then, the consultations were refined with online surveys embedded in them. These consultations are currently available online to collect more data.

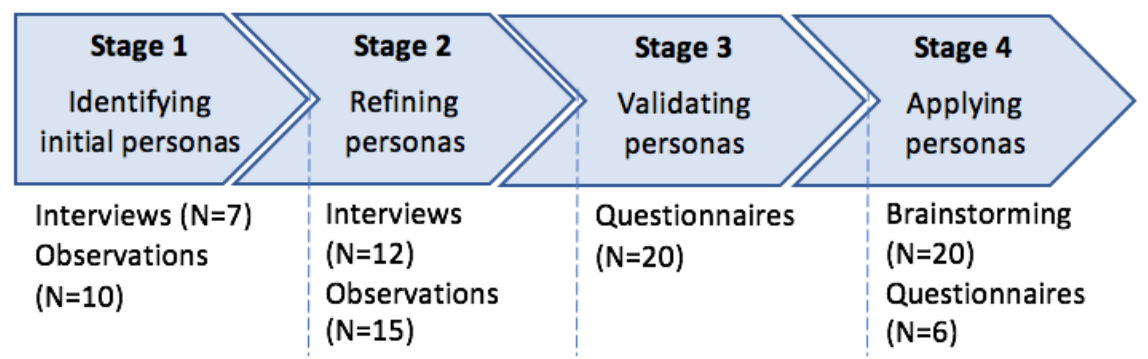

Figure 3: Project Stages 


\section{DATA ANALYSIS}

The transcriptions of the interviews and handwritten notes from observations were analysed in NVivo [17]. First, open coding was used to identify concepts in data [18]. These consist of a short phrase that captures the essence of a portion of text or visual data. The open coding was followed by axial coding where codes are grouped into conceptual categories [18]. A set of conceptual categories were derived from the persona's characteristics, such as motivation, goals, frustrations, challenges, background. Other categories came up from data (e.g. introduction to the model, and confidence). The data from the questionnaires were analysed using a method inspired from the thematic analysis [19].

\section{RESULTS}

Three personas were identified and described in detail.

RI Professional: Philip is committed to making his work for his RI accurate, reliable and effective. When changes are needed he balances helping with their implementation against meeting his commitments. He uses the RM to: 1) model the processes undertaken by his $\mathrm{RI}$; 2) find a common language to discuss how an Rl works to make interaction easier within and beyond his $\mathrm{Rl}$; 3) identify gaps, strengths and weaknesses in his Rl's systems and organisation; 4) address the gaps and weaknesses; 5) help his RI to develop; 6) improve interoperation with other Rls and provide external services. For example, an RI professional, working within the atmospheric domain stated that: "ENVRI $R M$ makes interaction a lot easier by offering a common language and a common understanding about how an RI works".

RI Systems Engineer: Edward needs to communicate well with his users and other stakeholders as he tries to meet their priorities, comply with regulations and negotiate for resources. He seeks good agreements for sustainable provision and use of ICT by his RI. Edward uses the RM to: 1) specify RI components in a standard language; 2) understand the $\mathrm{Rl}$ processes to achieve an interoperation platform with similar Rls. A software engineer declared that: "As a developer, I think it [ENVRI RM] is useful to understand my $R I$ and to bring an understanding of different R/s."

RI Strategist / Manager: Sabina is interested in building and improving her $\mathrm{Rl}$ and aligning it with other Rls. Her goals are to: 1) pool effort and improve return on investment; 2) develop a data management plan; 3) standardise data handling for multi-thematic domains. A manager from a marine research institution, affirmed that: "It is [ENVRI RM] a nice model/framework to help people build a coherent model; good for information management. It addresses the standardisation of data circulations from multi-thematic domains (for example space, ocean, atmosphere)".

\subsection{Challenges}

Two challenges were common to all personas: the lack of clear examples of the use of the model and the difficulty to allocate time while meeting their RI commitments.

The most important specific challenges for Philip (highlighted by all participants to the studies) were the lack of introductory materials and difficulties with the diagrams. For example, a marine RI professional declared that: "It [ENVRI RM] is too technical-based, especially the diagrams are difficult to be understood by non-IT people."

For Edward, the most challenging difficulty was to use the model from the point of view of implementation, as stated by one of the RI Systems Engineer from an atmospheric research institution: "It is difficult from the point of view of implementation. For example, I don't understand how to apply the model in engineering to derive an optimal architecture to help developers to build a kind of reasonable technological path to do the development".

Sabina's most challenging problems with the ENVRI RM were the difficulty to see the benefits for the short-term and the difficulty to cope with the size of the model, as it is illustrated in the quotes presented below: "We need to involve too much time and I don't see the benefits on short time" [an RI Strategist in a marine research institution]

\subsection{Personas' validation}

19 out of 20 of the participants at stage 3 identified themselves with a persona ( 3 with Philip, 11 with Edward and 5 with Sabina - which is the distribution at the meetings, not in the target community). The other participant named himself as a "software engineer", but he did not provide any additional information. A few additional goals and challenges were identified and added to the personas description.

\subsection{Suggestions for model communication}

The suggestions regarding the communication about the RM were classified into two categories: 1) raising the motivation and awareness, and; 2) improving the model presentation in terms of structure and language.

While all personas highlighted the need for more examples and for a clear guide to applying the RM, people identifying themselves with Philip made suggestions about simplifying the language for the $\mathrm{RM}$, avoiding the IT specific terms and using a textual replacement for the diagrammatic representations. A suggestion coming from this group was to create introductory material as an intellectual ramp [20], from general to detailed levels. 


\subsection{Feedback on consultations}

The feedback on the RM overview for the RI professional was positive from five out of the six participants. Only one participant considered it as being inappropriate, but he did not provide an explanation for that. A list of suggestions was collected to help improve the overview. That included: add concrete benefits for the RI professional, reference tools, languages, etc. that let them use the ENVRI RM. The results from the online surveys are not available yet.

\section{DISCUSSION}

The ENVRI RM models the "archetypical" environmental RI. It has been developed to assist $\mathrm{RI}$ systems design and to promote interoperability. There have been 6 reports of it being used successfully [9]. However, the RM has not been as widely adopted as expected, provoking the investigation reported in this paper. Moreover, people who were interviewed with four exceptions, did not feel confident yet in applying the model. We sought possible ways to increase adoption (without an expert trainer as that would not be sustainable) through improving the communication about the $\mathrm{RM}$. We used personas to refine our understanding of potential users. We conducted a series of studies involving 65 subjects to: 1) identify and describe personas related to the RM, including their goals, challenges and frustrations in relation to the ENVRI RM; 2) apply personas to characterise and overcome the impediments to adoption of the RM.

We identified 3 personas within the environmental RIs: the RI professional (Philip), the RI systems engineer (Edward) and the RI strategist/manager (Sabina). After refinement, the personas' descriptions were regarded as accurate by the majority of the RM users.

From each persona's description, Philip has the most challenges when using the RM because he does not have formal IT skills and is not familiar with Unified Modelling Language (UML) diagrams. The technical language of the ENVRI RM, its benefits in systems views of infrastructure and the systems-oriented thought processes needed to effectively exploit the RM are particularly challenging. A biologist pointed out: "It [the ENVRI $\mathrm{RM}$ ] is difficult to understand and handle; it uses specific technical language - easy for engineers, but difficult for others (e.g. RI researchers). [...] Diagrams are difficult to understand." Edward has in-depth knowledge about computing and distributed ICT systems, and of the technical challenges and trade-offs implicit in the architectural and technical design of such systems. Even so, individuals with this persona still find it difficult to apply the RM as they implement new RI components. They struggle with the connection between their existing RI systems and the RM. For
Sabina, the challenges are to see the short-term benefits and to cope with the size of the RM. A manager in a marine $\mathrm{RI}$ reported that: "there is a lot of content, too much to read about it [...] intimidating". As a consequence of these findings, different ways of presenting the ENVRI RM should be available for each persona.

Personas helped identify the level of support required by different users, and the different aspects of the RM that are important to different audiences. Thus, Philip, the RI professional needs more support to understand and apply the science and information viewpoints of the RM as this persona is more likely to be involved in discussions and planning of their RI from the science and information points of view. Edward, the RI systems engineer needs support in translating the science and information needs of the $\mathrm{RI}$ into computational, engineering and technology terms as he designs the architecture of the RI. Sabina, as the RI Strategist/ Manager needs help to keep an overview of all these aspects as she seeks to build, improve and align her RI with other RIs. Personas helped us discover these differences in their needs for the RM and enabled us to improve our promotion and communication about it. In addition, we used personas to decide what is the target audience to address first. That was Philip as he needs more support to understand and apply the RM. Sabina and Edward will be addressed in later work.

\section{CONCLUSIONS AND FUTURE WORK}

We investigated the following question: Can the persona technique be helpful for a specific reference model (i.e. ENVRI RM)? We have preliminary evidence based on one sample conceptual RM in one community that the persona technique is helpful. We made significant progress discovering how to improve and target communication about the complex information systems to each persona. This is just a preliminary step in investigating the value of persona for conceptual products.

Future work should evaluate the effectiveness of the consultations, after they are completed and made accessible. The persona technique should be used to answer more research questions, such as: "To what extent is the ENRI RM beneficial to each persona?"; "To what extent are the benefits of the RM perceived by each persona?", "What other personas exist outside the Rls and what do they use the RM for?" This should lead to further evidence of the value of the technique, as well as improved communication with such application - domain audiences about the systems they use or need.

\section{Acknowledgements}

This work was supported by [removed for blind review]. We thank all the participants in the studies and [removed for blind peer review] for their constructive feedback. 


\section{References}

1. ENVRI RM. 2017. Retrieved Apr. 2018 from http://envri.eu/rm

2. ESFRI. 2016. Retrieved Mar. 2018 from https://ec.europa.eu/research/infrastructures/pd f/esfri/esfri_roadmap/esfri_roadmap_2016_full. pdf

3. EUFAR. 2018. Retrieved Mar. 2018 from https://www.eufar.net

4. Linington, P. F., Milosevic, Z., Tanaka, A., Vallecillo, A. 2011. Building enterprise systems with ODP. CRC Press.

5. OASIS. 2006. Retrieved Apr. 2018 from https://docs.oasis-open.org/soa-rm/v1.0/soarm.html

6. Shames, P., Yamada, T. (2003). Reference architecture for space data systems.

7. Tanaka A., Miyazaki H. 2007. Experimental Development of UML Profile for Japanese Governement's Enterprise Architecture. INTAP.

8. Bureau of Meteorology. Draft, C. National Environmental Information Infrastructure Reference Architecture. Retrieved Apr. 2018 from:

http://www.bom.gov.au/environment/doc/neii_r eference_architecture-consultation_draft.pdf

9. Chen, Y., Hardisty, A. 2014. A common reference model for environmental RIs, iLEAPS newsletter. Retrieved Jan. 2018 from https://confluence.egi.eu/display/EC/ENVRI+R eference+Model

10. ENVRI RM technical training. 2017. Retrieved Apr. 2018 from https://training.envri.eu/course/index.php?categ oryid $=16$

11. Cooper, A., Reimann, R., Cronin, D. 2007. About Face 3: The essentials of interaction design. Wiley.
12. Cooper, A. 2004. The inmates are running the asylum: [Why high-tech products drive us crazy and how to restore the sanity]. Indianapolis: Sams.

13. Castro, J., Acua, S. Juristo, N. 2008. Integrating the personas technique into the requirements analysis activity. Computer Science, ENC'08, Mexican International Conference, 104-112.

14. Miaskiewicz, T., Kozar, K. A. 2011. Personas and user-centered design: How can personas benefit product design processes? Design Studies, 32(5): 417-430.

15. Switzky, A. 2012. Incorporating UCD into the software development lifecycle: a case study. In Proceedings of the $2012 \mathrm{CHI}$ Conference on Human Factors in Computing Systems, ACM, 469-484.

16. Matthews, T., Judge, T., Whittaker, S. 2012. How Do Designers and User Experience Professionals Actually Perceive and Use Personas? In Proceedings of the $2012 \mathrm{CHI}$ Conference on Human Factors in Computing Systems, ACM, 1219-1228.

17. QSRI. 2016. Retrieved Apr. 2018 from http://www.qsrinternational.com/

18. Saldana, J. 2013. The coding manual for qualitative researchers. CA: Sage.

19. Braun, V., \& Clarke, V. (2006). Using thematic analysis in psychology. Qualitative research in psychology, 3(2): 77-101.

20. Atkinson, M., De Roure, D., van Hemert, J., and Michaelides, D. 2010. Shaping ramps for data-intensive research. At UK e-Science All Hands Meeting 2010. Retrieved Apr. 2018 from https://eprints.soton.ac.uk/271235/. 